\title{
Asian Organization for Crohn's and Colitis and Asia Pacific Association of Gastroenterology consensus on tuberculosis infection in patients with inflammatory bowel disease receiving anti-tumor necrosis factor treatment. Part 1: risk assessment
}

Dong Il Park ${ }^{1}$, Tadakazu Hisamatsu', Minhu Chen ${ }^{3}$, Siew Chien $\mathrm{Ng}^{4}$, Choon Jin Ooi ${ }^{5}$, Shu Chen Wei ${ }^{6}$, Rupa Banerjee ${ }^{7}$ Ida Normiha Hilmi ${ }^{8}$, Yoon Tae Jeen ${ }^{9}$, Dong Soo Han ${ }^{10}$, Hyo Jong Kim ${ }^{11}$, Zhihua Ran ${ }^{12}$, Kaichun $\mathrm{Wu}^{13}$, Jiaming Qian ${ }^{14}$, Pin-Jin $\mathrm{Hu}^{3}$, Katsuyoshi Matsuoka ${ }^{15}$, Akira Andoh ${ }^{16}$, Yasuo Suzuki ${ }^{17}$, Kentaro Sugano $^{18}$, Mamoru Watanabe ${ }^{15}$, Toshifumi Hibi ${ }^{19}$, Amarender S. Puri ${ }^{20}$, Suk-Kyun Yang ${ }^{21}$ ${ }^{I}$ Department of Internal Medicine, Kangbuk Samsung Hospital, Sungkyunkwan University School of Medicine, Seoul, Korea, ${ }^{2}$ The Third Department of Internal Medicine, Kyorin University School of Medicine, Tokyo, Japan, ${ }^{3}$ Department of Gastroenterology, The First Affiliated Hospital, Sun Yat-sen University, Guangzhou, ${ }^{4}$ Department of Medicine and Therapeutics, Institute of Digestive Disease, LKS Institute of Health Science, State Key Laboratory of Digestive Disease, The Chinese University of Hong Kong, Hong Kong, China, ${ }^{5}$ Gleneagles Medical Centre and Duke-NUS Medical School, Singapore, ${ }^{6}$ Department of Internal Medicine, National Taiwan University Hospital, National Taiwan University College of Medicine, Taipei, Taiwan, ${ }^{7}$ Department of Medical Gastroenterology, Asian Institute of Gastroenterology, Hyderabad, India, ${ }^{8}$ Department of Medicine, University of Malaya, Kuala Lumpur, Malaysia, ${ }^{9}$ Department of Internal Medicine, Korea University College of Medicine, Seoul, ${ }^{10}$ Department of Internal Medicine, Hanyang University Guri Hospital, Guri, ${ }^{11}$ Department of Internal Medicine, Kyung Hee University School of Medicine, Seoul, Korea, ${ }^{12}$ Department of Gastroenterology, Shanghai Jiao Tong University, Shanghai, ${ }^{13}$ Department of Gastroenterology, Fourth Military Medical University, Xian, ${ }^{14}$ Department of Gastroenterology, Peking Union Medical College, Beijing, China, ${ }^{15}$ Department of Gastroenterology and Hepatology, Tokyo Medical and Dental University, Tokyo, ${ }^{16}$ Department of Gastroenterology, Shiga University, Otsu, ${ }^{17}$ Department of Internal Medicine, Toho University, Sakura, ${ }^{18}$ Department of Medicine, Jichi Medical University, Shimotsuke, ${ }^{19}$ Center for Advanced IBD Research and Treatment, Kitasato University, Tokyo, Japan, ${ }^{20}$ Department of Gastroenterology, Govind Ballabh Pant Institute of Postgraduate Medical Education and Research, New Delhi, India, ${ }^{21}$ Department of Gastroenterology, Asan Medical Center, University of Ulsan College of Medicine, Seoul, Korea

Because anti-tumor necrosis factor (anti-TNF) therapy has become increasingly popular in many Asian countries, the risk of developing active tuberculosis (TB) among anti-TNF users may raise serious health problems in this region. Thus, the Asian Organization for Crohn's and Colitis and the Asia Pacific Association of Gastroenterology have developed a set of consensus statements about risk assessment, detection and prevention of latent TB infection, and management of active TB infection in patients with inflammatory bowel disease (IBD) receiving anti-TNF treatment. Twenty-three consensus statements were initially drafted and then discussed by the committee members. The quality of evidence and the strength of recommendations were assessed by using the Grading of Recommendations Assessment, Development, and Evaluation methodology. Webbased consensus voting was performed by 211 IBD specialists from 9 Asian countries concerning each statement. A consensus statement was accepted if at least $75 \%$ of the participants agreed. Part 1 of the statements comprised 2 parts: risk of TB infection

Received October 8, 2017. Revised October 12, 2017. Accepted October 13, 2017. Published online November 9, 2017

Correspondence to Suk-Kyun Yang, Department of Gastroenterology, Asan Medical Center, University of Ulsan College of Medicine, 88 Olympic-ro $43-$ gil, Songpagu, Seoul 05505, Korea. Tel: +82-2-3010-3901, Fax: +82-2-476-0824, E-mail: sky@amc.seoul.kr

These consensus were developed and approved by the AOCC and APAGE, and are being published simultaneously in the Intestinal Research and Journal of Gastroenterology and Hepatology.

\footnotetext{
(c) Copyright 2018. Korean Association for the Study of Intestinal Diseases. All rights reserved.

This is an Open Access article distributed under the terms of the Creative Commons Attribution Non-Commercial License (http://creativecommons.org/licenses/by-nc/4.0)

which permits unrestricted non-commercial use, distribution, and reproduction in any medium, provided the original work is properly cited.
} 
during anti-TNF therapy, and screening for TB infection prior to commencing anti-TNF therapy. These consensus statements will help clinicians optimize patient outcomes by reducing the morbidity and mortality related to TB infections in patients with IBD receiving anti-TNF treatment. (Intest Res 2018;16:4-16)

Key Words: Tuberculosis; Anti-tumor necrosis factor; Inflammatory bowel disease; Consensus statement

\section{INTRODUCTION}

Approximately one-third of the worldwide population is estimated to be infected with Mycobacterium tuberculosis (MTB). ${ }^{1}$ Of these infected individuals, $10 \%$ develop an active tuberculosis (TB) infection and the remaining 90\% develop a latent TB infection (LTBI), characterized by the presence of an immune response against MTB despite the absence of signs or symptoms of TB disease. ${ }^{2}$ However, LTBI may progress to active TB if there is an imbalance in the host immune regulation caused by human immunodeficiency virus infection, malnutrition, malignancy, or treatment with immunosuppressive agents such as anti-tumor necrosis factor (antiTNF) agents. TNF- $\alpha$ plays a central role in the host defense against MTB, particularly in the formation and maintenance of granulomas, limiting the dissemination of infection., Therefore, the inhibition of TNF- $\alpha$ can increase the susceptibility to MTB and accelerate the reactivation of LTBI. ${ }^{5}$

Anti-TNF therapy initiated a new therapeutic era against chronic immune-mediated inflammatory diseases such as IBD and is recommended as the standard care for patients who fail to respond to conventional treatment in countries where the health-care system can afford its high cost. ${ }^{6}$ However, shortly after the launch of anti-TNF treatment, the incidence of active TB increased among anti-TNF users throughout North America and Europe. ${ }^{7}$ Previous studies based on the national registry of anti-TNF recipients also revealed an increased risk of TB in patients who were treated with anti-TNF agents. ${ }^{8,9}$ Most cases developed within several months after the initiation of anti-TNF therapy, suggesting a reactivation of LTBI; however, delayed cases consistent with new infections have been occasionally reported. ${ }^{7}$ The incidence of TB in patients with IBD receiving anti-TNF therapy may vary depending on the prevalence of $\mathrm{TB}$ in the general population, type of anti-TNF agent used, and type of underlying disease. ${ }^{10}$ Because anti-TNF therapy has become increasingly popular in many Asian countries, where the prevalence of LTBI is much higher than that in Western countries, the risk of active TB among anti-TNF users may raise serious health problems in this region. ${ }^{11,12}$ The incidence of TB among anti-TNF users has markedly decreased owing to routine LTBI screening and treatment in potential anti-TNF users. ${ }^{13}$ Therefore, many scientific societies and national public health agencies currently recommend that all potential anti-TNF users should be screened for LTBI. ${ }^{14-28}$ Moreover, this need for screening is required to a greater extent in many Asian countries, where the prevalence of LTBI is higher, than in Western countries. However, many questions remain unanswered concerning the need for screening, the best diagnostic approach, and preventive measures of LTBI prior to anti-TNF therapy, as well as concerning the appropriate management of active TB and the resumption of anti-TNF therapy after TB treatment. For this reason, the Asian Organization for Crohn's and Colitis and the Asia Pacific Association of Gastroenterology developed a set of consensus statements on the risk assessment, detection and prevention of LTBI, and management of active TB infection in patients with IBD receiving anti-TNF treatment. These recommendations will help clinicians optimize patient outcomes by reducing the morbidity and mortality associated with TB infection.

\section{METHODS}

The process and procedures for the development of consensus statements complied with the World Health Organization Guidelines Review Committee requirements, including the establishment of a guideline development panel, a systematic review of the evidence, and the formulation of recommendations by using a structured process. ${ }^{29}$ After data appraisal, 23 consensus statements were initially drafted by D.I.P. and S.K.Y., and then discussed by the committee members; furthermore, the evidence was debated and the statements were redrafted several times. Although the draft was primarily based on published evidence, in some areas where the level of evidence was very low, reflecting the paucity of randomized controlled trials, expert opinions were included where required. Part 1 of the statements comprised 2 parts: (1) risk of TB infection during anti-TNF therapy, and (2) screening for TB infection prior to anti-TNF therapy.

The quality of evidence and the strength of recommendations were assessed by using the Grading of Recommenda- 
tions Assessment, Development, and Evaluation (GRADE) methodology when applicable..$^{30}$ In the GRADE process, the quality of evidence of each statement was categorized as high, moderate, low, or very low. Evidence based on randomized controlled trials was initially classified as highquality evidence but could be downgraded for several reasons, including study limitations, inconsistency of results, indirectness of evidence, imprecision, and reporting bias. Although data from observational studies (e.g., cohort and case-control studies) were initially classified as low-quality evidence, the rating could be upgraded if the magnitude of the treatment effect was substantial, if there was evidence of a dose-response relationship, or if all plausible biases were found to decrease the magnitude of an apparent treatment effect. ${ }^{30,31}$ On the basis of the GRADE system, the strength of recommendations was classified as either strong or weak, as determined on the basis of 4 key factors: (1) quality of evidence; (2) balance of desirable benefits and undesirable harm; (3) values and preferences of clients and health-care providers; and (4) resource implications. ${ }^{32}$

Web-based consensus voting was performed by 211 IBD specialists from 9 Asian countries concerning each statement. A consensus statement was accepted if at least $75 \%$ of the participants voted 1 (strongly agree) or 2 (agree) on a scale of 1 to 5 (with 3, 4, and 5 indicating uncertain, disagree, and strongly disagree, respectively). If a statement was not accepted, the wording of the statement was discussed and revised, and then re-voting was conducted. ${ }^{33}$

\section{RISK OF TB INFECTION DURING ANTI-TNF THERAPY}

Reactivation of latent TB is increased in patients with IBD receiving anti-TNF treatment

- Quality of evidence, moderate; recommendations, strong

- Level of agreement: strongly agree 51\%, agree 39\%, uncertain $7 \%$, disagree $3 \%$

Although anti-TNF therapies have revolutionized the treatment of chronic immune-mediated inflammatory diseases, including IBD, these agents have been associated with a 2- to 8 -fold increased risk of active TB in these patients compared with that in the general population. ${ }^{34-37}$ Previous studies based on a national registry also revealed that anti-TNF therapy is associated with a nearly 4 -fold increased risk of developing TB. ${ }^{19,38}$ Because not all cases are reported, the actual risk is suspected to be higher than expected. In a systematic review of 40 randomized controlled trials with a total of
14,683 patients, the incidence of TB was $0.26 \%(26 / 10,010)$ in the anti-TNF group and $0 \%(0 / 4,673)$ in the control group receiving a placebo or placebo plus immunosuppressant, corresponding to an OR of 24.8 (95\% CI, 2.4-133.0; $P<0.01){ }^{24}$ The risk of developing active TB was even greater when antiTNF was combined with another immunosuppressant when compared with the control (OR, 54.0; 95\% CI, 5.3-88.0) or anti-TNF monotherapy (OR, 13.3; 95\% CI, 3.7-100.0) group. ${ }^{24}$ According to a meta-analysis of randomized controlled trials for anti-TNF therapy in patients with IBD, the risk of TB infection among patients allocated to the anti-TNF therapy group increased 2.52-fold over that in those who received the placebo (95\% CI, 0.62-10.21); however, the difference was not statistically significant. ${ }^{39}$

The risk of developing TB in patients receiving anti-TNF therapy may vary depending on the type of underlying disease and actual anti-TNF agent used. ${ }^{8,40,41}$ Among 8,421 patients who were prescribed anti-TNF therapy in a Korean national database, ${ }^{40}$ the incidence of TB (events per $10^{5}$ personyears) was the highest in patients with $\operatorname{IBD}(3,710)$, followed by rheumatoid arthritis $(1,143)$, psoriatic arthritis (934), and ankylosing spondylitis (715). The risk of developing TB may vary according to the specific anti-TNF agent, with higher risks reported with the use of the monoclonal antibodies infliximab and adalimumab relative to those reported with the use of the soluble TNF-receptor antagonist etanercept. ${ }^{8,41}$

Most of the active TB cases occurred within 3 to 4 months after the initiation of anti-TNF therapy. Thus, reactivation of LTBI rather than a new infection is considered to be the primary cause. ${ }^{34}$ For these reasons, screening for LTBI before initiating anti-TNF therapy is strongly recommended by many scientific organizations and health authorities worldwide. $^{14-28}$

The incidence rate of TB reactivation among patients with IBD undergoing anti-TNF therapy is higher in TB-endemic areas, including many Asian countries

- Quality of evidence, low; recommendations, weak

- Level of agreement: strongly agree 34\%, agree 48\%, uncertain $17 \%$, disagree $1 \%$

Most new TB cases occur in Asia (59\%) and Africa (26\%), with a smaller proportion of cases occurring in Europe (5\%) and North America (3\%). ${ }^{42}$ It has been estimated that almost one-third of the global population has LTBI. Moreover, LTBI is a growing concern, particularly in TB-endemic areas. For patients with IBD, the current incidence of active TB during anti-TNF therapy is approximately $1 \%$ to $2 \%{ }^{43,44}$ The risk 
of developing active TB in patients with LTBI is increased during anti-TNF therapy even after LTBI screening and treatment. ${ }^{43}$ In TB-endemic areas, the risk of developing TB is also elevated by close contact with infectious patients with TB.

It has been suggested that the risk of developing TB among patients with IBD undergoing anti-TNF therapy might be substantially higher in TB-endemic areas, including many Asian countries, than in Western Europe or North America. ${ }^{10}$ Comparing the nationwide registry databases of patients receiving anti-TNF for IBD and rheumatologic diseases, the reported number of TB cases per $10^{5}$ person-years was 144 in the United States, ${ }^{7} 130$ in United Kingdom, ${ }^{41} 117$ in France, ${ }^{8}$ 230 in Spain, ${ }^{35}$ and 690 in Turkey. ${ }^{45}$ In a nationwide database from South Korea, including 8,421 patients treated with an anti-TNF agent, the incidence of TB was 1,017 per $10^{5}$ person-years. ${ }^{40}$ In 4 retrospective cohort studies, including patients with IBD receiving anti-TNF treatment, conducted in South Korea, ${ }^{46,47}$ Taiwan, ${ }^{48}$ and Hong Kong, ${ }^{49}$ the number of reported TB cases per $10^{5}$ person-years was $1,997,{ }^{46} 2,484,{ }^{47}$ $1,900,{ }^{48}$ and $4,938,{ }^{49}$ respectively, per $10^{5}$ person-years.

The risk of TB reactivation is higher when anti-TNF agents are combined with immunosuppressive agents

- Quality of evidence, moderate; recommendations, strong

- Level of agreement: strongly agree 35\%, agree 43\%, uncertain $20 \%$, disagree $2 \%$

In a systematic review of 40 randomized controlled trials, including 10,010 patients with anti-TNF therapy and 4,673 patients administered a placebo, TB reactivation was increased by 24.8 -fold in patients who received anti-TNF therapy as compared with that in the control group $(26 / 10,010$ vs. 0/4,673; OR, 24.8; 95\% CI, 2.4-133.0; $P<0.001) .{ }^{24}$ Moreover, TB reactivation increased 54 -fold in patients treated with a combination of anti-TNF and immunosuppressive agents as compared with that in the control group $(24 / 4,241 \mathrm{vs.}$ 0/4,673; OR, 54.0; 95\% CI, 5.3-88.0; $P<0.001)$. TB reactivation also increased 13.3-fold in patients treated with a combination of anti-TNF and immunosuppressive agents compared with that in those with anti-TNF monotherapy (24/4,241 vs. 2/5,769; OR, 13.3; 95\% CI, 3.7-100.0; $P<0.001)$. It was suggested that the additional TB risk was related to a synergistic effect between the anti-TNF agents and methotrexate or azathioprine rather than the intrinsic risk of each immunosuppressive drug because the simple addition of immunosuppressive agents to the placebo group did not increase the risk of developing TB.

In another study using the Food and Drug Administration
Adverse Event Reporting System between January 2003 and June 2011, the authors searched for "Primary Suspect" reports of various infections associated with anti-TNF, systemic corticosteroids, and immunosuppressive agents with a usage indication for IBD. ${ }^{50}$ When analyzing TB infections, anti-TNF monotherapy was associated with an 8.5-fold increased risk of TB infection compared with 5 -aminosalicylic acid therapy (OR, 8.52; 95\% CI, 1.96-37.01; $P<0.001$ ). Combination therapies, including anti-TNF agents with an immunosuppressant (OR, 25.27; 95\% CI, 5.66-112.72; $P<0.001)$ or a systemic corticosteroid (OR, 3.17; 95\% CI, $0.22-46.76$; $P=0.42$ ) or both (OR, 24.28; 95\% CI, 5.29-111.43; $P<0.001)$, were similarly associated with an increased risk of TB infection compared with 5-aminosalicylic acid therapy. Therefore, more intensive LTBI screening and surveillance programs should be recommended for patients receiving combined anti-TNF agents and immunosuppressant and/or systemic corticosteroids.

Negative screening results do not exclude the risk of TB infection in patients with IBD receiving anti-TNF therapy

- Quality of evidence, low; recommendations, weak

- Level of agreement: strongly agree 19\%, agree 60\%, uncertain $16 \%$, disagree $5 \%$

In 2 retrospective cohort studies from Spain ${ }^{43}$ and Portugal, ${ }^{51}$ as well as in a case report from India, ${ }^{52}$ negative LTBI screening results from chest radiography and tuberculin skin test (TST) could not exclude the risk of TB infection in patients with IBD and rheumatologic diseases receiving anti-TNF therapy. Of 423 patients with IBD treated with antiTNF, 7 (1.65\%) developed TB during anti-TNF therapy. ${ }^{43}$ Among the 7 patients who developed TB, 6 had negative LTBI screening results and 4 developed TB within the first 16 weeks after anti-TNF therapy initiation. Of 765 patients with rheumatologic diseases taking anti-TNF, 25 patients were diagnosed as having active $\mathrm{TB} .{ }^{51}$ Among the 17 patients tested for latent TB, 13 had negative TST results. These findings suggest that living in a region with high LTBI prevalence and concomitant immunosuppressant use, along with the low sensitivity of TST, may correspond to false-negative results in the LTBI screening protocol.

In an integrated analysis of LTBI screening data from 5 large phase III trials of golimumab in patients with rheumatologic diseases, 2,282 patients underwent both an interferon-gamma releasing assay (IGRA) and TST screening prior to golimumab treatment. ${ }^{53}$ Among these patients, $13.8 \%$ had LTBI, including 9.4\% with positive TST results, 7.0\% with positive results in the QuantiFERON-TB Gold In-Tube 
test (QFT-GIT; Cellestis, Carnegie, Australia), and 2.6\% with positive results in both tests. Among the patients who had negative results in both TST and QFT-GIT during screening, 5 developed active TB during the 1-year follow-up. In a retrospective descriptive study conducted at 20 French and Swiss centers on all patients with IBD undergoing anti-TNF therapy who developed TB despite negative initial LTBI screening test results (thorough history and clinical examination, TST and/or QFT-GIT, chest radiography, or chest CT), 44 TB cases were identified. ${ }^{54}$ Among the 6 patients who experienced a reactivation of LTBI (active TB diagnosed within the first 3 months), 4 patients underwent only TST and 2 were tested with only QFT-GIT. In a prospective study that included 426 patients with immune-mediated inflammatory diseases, both TST and QFT-GIT were performed before commencing anti-TNF therapy. ${ }^{55}$ During a median of 297 days of followup, active TB developed in 1.4\% (6/426) of the patients who tested negative for TST and QFT-GIT at baseline. In a retrospective cohort study of all TB cases identified out of 873 patients with IBD receiving anti-TNF therapy, 19 of 25 new TB cases developed despite negative LTBI screening with IGRA. ${ }^{47}$ Therefore, we can reasonably conclude that negative screening results do not exclude the risk of TB infection in patients with IBD undergoing anti-TNF therapy. Based on the above facts, it is logical to suggest that all IBD patients who start anti-TNF therapy need to be closely monitored for reactivation of TB irrespective of the outcome of the screening tests.

\section{SCREENING FOR TB INFECTION PRIOR TO ANTI- TNF THERAPY}

Screening for latent or active TB should always be performed prior to commencing anti-TNF treatment

- Quality of evidence, moderate; recommendations, strong

- Level of agreement: strongly agree 91\%, agree 9\%

Systematic screening for LTBI or active TB should be considered at the time of initial diagnosis and always performed prior to commencing anti-TNF therapy. The introduction of LTBI screening protocols to candidate patients for antiTNF therapy has had a beneficial impact on the incidence of active TB among Spanish patients with rheumatologic diseases. Patients exhibited a 21-fold higher risk of developing active TB compared with the general Spanish population before preventive actions were proposed. After the adoption of official recommendations, however, the development of active TB decreased by $78 \% .^{13}$ The strict recommendation of chemoprophylaxis for LTBI has reduced the incidence of new $\mathrm{TB}$ cases among infliximab users from 11 patients in the first 2,000 infliximab users to only 2 patients in the second 2,000 registrants. ${ }^{56}$ The incidence of TB infection associated with anti-TNF therapy is higher in real-life clinical data than in controlled trials owing to the poor compliance with LTBI screening protocols. In a retrospective study performed at a large urban academic hospital in the United States, only 65\% of patients were screened for LTBI prior to the initiation of anti-TNF therapy, and risk factors for TB were documented in only $17 \% .{ }^{57}$ Moreover, in a retrospective cohort study from South Korea, 18.5\% of patients (161/873) did not undergo systematic screening for LTBI prior to initiation of anti-TNF therapy, and even in patients diagnosed with LTBI, a substantial proportion $(13.7 \%, 10 / 73)$ did not receive prophylactic therapy. ${ }^{47}$

Screening for LTBI should be performed in patients before treatment with immunosuppressive drugs to avoid falsenegative and inconclusive results. TST and the newer IGRA tests, including QFT-GIT and T-SPOT (T-SPOT.TB; Oxford Immunotec, Abingdon, UK), are often associated with falsenegative and false-positive results, particularly in immunocompromised patients taking immunosuppressants or anti-TNF agents. ${ }^{53}$ A previous study reported that the falsenegative rate of TST reached as high as $40 \%$ in patients with rheumatoid arthritis. ${ }^{58}$ In a large-scale meta-analysis of 124 studies, the pooled rate of the indeterminate results of QFTGIT and T-SPOT increased from 2.1\% (95\% CI, 0.020-0.023) to $4.4 \%$ (95\% CI, 0.039-0.050) and from 3.8\% (95\% CI, $0.035-0.042)$ to $6.1 \%$ (95\% CI, 0.052-0.071), respectively, in immunocompromised patients. ${ }^{59}$

Latent TB is diagnosed on the basis of prior history of TB treatment and contact with patients with $T B$, chest radiography, TST, and/or IGRAs. There are local variations in the recommendations for utilizing these modalities

- Quality of evidence, high; recommendations, strong

- Level of agreement: strongly agree 55\%, agree $42 \%$, uncertain $2 \%$, disagree $1 \%$

Currently, there is no "gold standard" for the diagnosis of LTBI. International guidelines recommend a TB risk evaluation prior to commencing anti-TNF therapy. This evaluation is based on epidemiological risk factors (Table 1), physical examination, chest radiography, TST, and/or IGRA; however, there are local variations in the recommendations for utilizing these modalities. ${ }^{14-28}$ A diagnosis of LTBI should be 
Table 1. Epidemiological Risk Factors for Latent TB Infection

Close contact with individuals known or suspected to have TB (family
members or persons sharing living spaces)
History of active TB or radiologic findings suggestive of past TB that
was not adequately treated
Living in or traveling to communities with high rates of latent or
active TB
Low-income populations
Residents of long-term care and correctional facilities
Occupational exposure to high-risk groups (health-care workers)
Close contact with individuals known to have human
immunodeficiency virus infection
Previous use of immunosuppressive drugs
Underlying diseases that predispose to MTB reactivation such as
diabetes, cirrhosis, and alcoholism

TB, tuberculosis; MTB, Mycobacterium tuberculosis.

considered if any of the following criteria are satisfied; recent exposure to patients with active TB, positive initial or booster TST and/or positive IGRA; and no radiological evidence of active TB. ${ }^{15}$

Acquiring patient history includes obtaining treatment history and data about previous medications used for active TB or LTBI, history of BCG vaccination, and data on current symptoms of suspected TB (e.g., cough, hemoptysis, fever, night sweats, weight loss, chest pain, shortness of breath, and fatigue of $>2$ weeks duration) before being tested for LTBI. A physical examination should be conducted, specifically targeting the parts of the body affected by active TB. The purpose of performing a chest radiography is to confirm the absence of active TB and the presence of old healed lesions (calcification $>5 \mathrm{~mm}$, pleural thickening, linear opacities, or upper lobe fibronodular disease) with no history of anti-TB treatment. ${ }^{60}$ Furthermore, Japanese guidelines recommend performing a chest CT in individuals with a high possibility of developing TB because minute lesions are occasionally detected on CT even when there are no abnormalities on a plain chest radiograph. ${ }^{18}$

In general, a TST should be considered positive if the induration is $\geq 5 \mathrm{~mm}$; however, there are local variations (Table 2). A cutoff of $10 \mathrm{~mm}$ may be considered to reduce false positives in patients with no epidemiologic risk factors, but this decision should be individualized. Moreover, TST results can be distorted by a prior BCG vaccination because vaccinated individuals may become positive reactors to purified protein derivate. Therefore, different cutoffs are recommended for BCG-vaccinated $(\geq 10 \mathrm{~mm})$ and nonvaccinated $(\geq 5 \mathrm{~mm})$
Table 2. Positive Cutoff Values of the Tuberculin Skin Test

\begin{tabular}{ll}
\hline \multicolumn{1}{c}{ Positive cutoff value } & \multicolumn{1}{c}{ Country } \\
\hline$\geq 5 \mathrm{~mm}$ & Brazil, Italia, Spain, Canada \\
$\begin{array}{l}\geq 10 \mathrm{~mm} \text { in BCG-vaccinated and } \geq 5 \mathrm{~mm} \\
\text { in BCG nonvaccinated }\end{array}$ & China, Taiwan \\
$\geq 10 \mathrm{~mm}$ in the general population and & Taiwan \\
$\geq 5 \mathrm{~mm}$ in immunocompromised & \\
$\geq 10 \mathrm{~mm}$ & South Korea, France, Japan \\
$\geq 15 \mathrm{~mm}$ in the general population and & Saudi Arabia \\
$\geq 10$ or $\geq 5$ mm in immunocompromised & \\
$>15 \mathrm{~mm}$ in BCG-vaccinated and $>6 \mathrm{~mm}$ & United Kingdom \\
in BCG nonvaccinated & \\
\hline
\end{tabular}

individuals in some countries (Table 2). This distortion is almost insignificant in adults aged $>30$ years, irrespective of the age at vaccination or revaccination. ${ }^{15}$ TST may also be negative in patients who have been taking corticosteroids for $>1$ month or thiopurines or methotrexate for $>3$ months. Consequently, a booster TST may be appropriate for patients taking immunomodulators with a negative TST 1 to 2 weeks after the first test. ${ }^{15}$

Recently, IGRA (i.e., QFT-GIT and T-SPOT) has become commercially available in many countries. GFT-GIT and TSPOT use purified antigens from MTB to stimulate peripheral blood lymphocytes for producing interferon- $\gamma$ (IFN- $\gamma$ ). The QFT-GIT test measures the amount of IFN- $\gamma$ in the supernatant of a cell suspension, whereas the T-SPOT test determines the number of cells producing IFN- $\gamma$ with the use of an ELISpot assay. IGRA is increasingly being used for the diagnosis of LTBI owing to its higher specificity and sensitivity, particularly in immunocompromised hosts. ${ }^{61,62}$ Moreover, IGRA does not exhibit any cross-reactivity with the BCG vaccine, which is another advantage of this method. Therefore, IGRAs may be particularly valuable for evaluating the TB infection status of individuals who had received a BCG vaccination.

Recent guidelines have changed the outlook concerning the diagnosis of LTBI. For example, the U.S. guidelines recommend replacing TST with IGRA as the diagnostic test for LTBI in all patients, and others recommend using both TST and IGRA either concomitantly or consecutively ${ }^{63-65}$ In addition, a recent publication from the United Kingdom verified that the LTBI detection rate was markedly increased when all 3 methods (clinical factors, TST, and IGRA) were used in combination. ${ }^{66}$ 


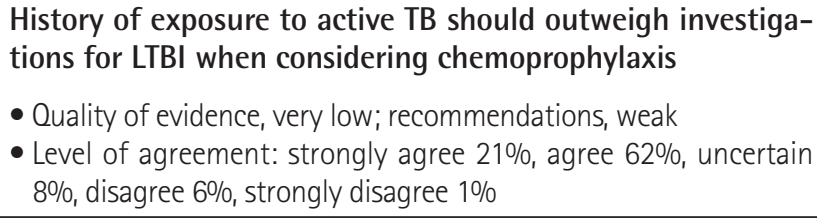

Most of the international guidelines strongly recommend evaluating the risk of an MTB infection by taking an accurate clinical history including recent or past close contacts with patients with $\mathrm{TB}$, being born in or having traveled to TB-endemic areas, being institutionalized, taking immunosuppressive drugs, and having underlying diseases associated with a predisposition to TB reactivation (e.g., diabetes, cirrhosis, and alcoholism) prior to initiating anti-TNF therapy. ${ }^{14-20,22,23,25-28}$ In the RATIO registry, patients born in TB-endemic areas had a 10.3-fold higher risk of developing TB after anti-TNF therapy. ${ }^{8}$ In a case report on a patient with UC who developed miliary TB after a second infusion of infliximab despite exhibiting a normal chest radiograph and negative IGRA test prior to starting infliximab, the patient had a history of very close contact with a patient with active pulmonary TB. ${ }^{67} \mathrm{An}$ other case of miliary TB was reported in a patient with IBD after 9 months of anti-TNF therapy despite several negative TSTs and indeterminate IGRA tests; however, the patient had a history of imprisonment for 4 years prior to the initiation of anti-TNF therapy. ${ }^{68}$ In a prospective study comparing the predictive value of IGRA and TST for the progression of LTBI to active TB, 6 of 601 contacts progressed to TB disease within a 2-year follow-up period. ${ }^{69}$ In a single-center cohort study from a TB-endemic country, taking a careful history of contact alone corresponded to 14 of 66 LTBI diagnoses $(21 \%))^{70}$

TST and IGRA tests are associated with false-negative results, especially in immunocompromised patients taking immunosuppressants or anti-TNFs. ${ }^{53,58}$ In a large-scale metaanalysis, the pooled rate of indeterminate results of IGRA was increased 2-fold in immunocompromised patients. ${ }^{59}$ There is a significant false-negative rate associated with TST and IGRA; thus, a negative result does not rule out LTBI. Patients with a clear history of a recent or past exposure to active TB were also candidates for LTBI treatment. Therefore, if a clear history of recent or past close exposure to $\mathrm{TB}$ is obtained, chemoprophylaxis should be performed before initiating anti-TNF therapy.
Abnormal chest radiographs suggestive of old TB in patients without a history of treatment should be considered as LTBI after excluding active TB

- Quality of evidence, very low; recommendations, weak

- Level of agreement: strongly agree $20 \%$, agree $57 \%$, uncertain $16 \%$, disagree $7 \%$

All guidelines recommend taking a chest radiograph, particularly in groups at a high-risk for TB development, except for the American College of Rheumatology, which recommends taking a chest radiograph only in case of positive TB infection tests (TST and IGRA) and the presence of risk factors. ${ }^{71}$ The purpose of obtaining a chest radiograph is to confirm the absence of active $\mathrm{TB}$ and the presence of old healed lesions (calcification $>5 \mathrm{~mm}$, pleural thickening, linear opacities, or upper lobe fibronodular disease) with no previous anti-TB treatment. ${ }^{60}$ The relative risk of developing active TB in individuals with untreated old TB lesions is considered 6 to 19 times higher than that in persons with no known risk factors, which is the highest risk after advanced human immunodeficiency virus infection and close contact with infectious $\mathrm{TB}^{72}$ In a single-center cohort study from a TB-endemic country, chest radiography was an important test for providing additional evidence of LTBI because $9 \%$ of the positive screening results were exclusively due to an abnormal chest radiograph. ${ }^{70}$ For individuals with untreated old TB lesions (except those with only pleural adhesion images or small calcification), prophylactic treatment with isoniazid (INH) for 24 weeks was reported to reduce the incidence of TB development by $65 \%{ }^{73}$ therefore, LTBI treatment has been determined to be useful for untreated old pulmonary TB. However, the presence of small $(\leq 5 \mathrm{~mm})$ calcified pulmonary nodules alone does not merit LTBI treatment because these lesions rarely display viable organisms upon an autopsy study. ${ }^{74}$ LTBI treatment is not indicated for patients with remaining fibrotic lesions if they had a history of adequate treatment for previous TB.

IGRAs are preferred over TST in BCG-vaccinated individuals, because TST exhibits cross-reactivity with the BCG vaccine, yielding false-positive results, whereas IGRAs do not

- Quality of evidence, high; recommendations, strong

- Level of agreement: strongly agree $45 \%$, agree $45 \%$, uncertain $10 \%$

It is important to recognize that the sensitivity of TST is sufficient; however, its specificity for predicting LTBI is not because only about $5 \%$ of immunocompetent patients with 
a positive TST result may progress from LTBI to active TB disease in their lifetime. ${ }^{75}$ A diagnosis of LTBI with TST may particularly be distorted by prior BCG vaccination because the purified protein derivatives used in the TST include antigens present in BCG strains, thereby leading to high false-positive results. ${ }^{15}$ However, this distortion is almost insignificant in adults aged $>30$ years, irrespective of the age at vaccination or revaccination ${ }^{15}$ because the frequency of a positive TST result is considerably reduced 2 years after vaccination, whereas no influence was observed after 10 years. $^{76,77}$

The high false positivity of TST induced by prior BCG vaccination may be overcome through the use of 2 new IGRAs (GFT-GIT and T-SPOT), which detect T-cell-mediated IFN- $\gamma$ responses to 2 specific MTB antigens (ESAT- 6 and CFP10), ${ }^{14}$ which are absent in the BCG strain. Therefore, IGRAs may be particularly valuable for evaluating the LTBI status of individuals who had been vaccinated with BCG. ${ }^{14}$

Multiple studies, particularly in immunocompetent patients, have demonstrated that IGRA tests are more sensitive and specific than TST, ${ }^{59,78,79}$ and their predictive value for the progression of LTBI to active TB disease has been demonstrated to be higher than that of TST, as observed in subjects with recent close contacts with patients with active $\mathrm{TB}^{69}$

Both IGRAs and TST can provide false-negative results in patients receiving immunosuppressive treatment; however, IGRAs are less influenced by immunosuppressive medications than is TST

- Quality of evidence, low; recommendations, weak

- Level of agreement: strongly agree 26\%, agree 67\%, uncertain $6 \%$, disagree $1 \%$

Immunosuppressive therapy (chronic systemic steroids, azathioprine, 6-mercaptopurine, methotrexate) in patients with IBD may reduce the sensitivity, thereby increasing the false-negative results of TST by inducing anergy and further resulting in a lack of a delayed-type hypersensitivity reaction. $^{80-82}$ This limitation diminishes the ability of clinicians to rely on TST as an adequate screening method for LTBI and reinforces the importance of the proper interpretation of TST results based on a patient's risk factors.

IGRA is a more specific and sensitive test for the diagnosis of LTBI than TST in immunocompetent patients. ${ }^{83}$ However, IGRA is also negatively influenced by immunosuppressive therapy. ${ }^{84}$ Ferrara et al. ${ }^{85}$ reported that patients receiving at least 1 immunosuppressive drug were three times more likely to have an indeterminate IGRA result than those not receiving such treatment (OR, 3.35; 95\% CI, 1.84-6.08; $P<0.0001)$. In a prospective case-control study, ${ }^{86}$ patients with IBD receiving immunosuppressive therapy were associated with a significantly lower IGRA positivity rate than those not receiving the therapy ( $13.0 \%$ vs. $29.6 \%, P=0.002)$. This difference seemed most prominent in patients taking azathioprine ( $11.8 \%$ vs. $27.3 \%, P=0.006$ ).

Both IGRA results (pooled OR, 0.37; 95\% CI, 0.16-0.87; $P=0.02$ ) and positive TST results (pooled OR, $0.28 ; 95 \% \mathrm{CI}$, $0.10-0.80 ; P=0.02$ ) are significantly influenced by immunosuppressive therapy; ${ }^{79}$ however, IGRAs are less influenced by immunosuppressive medications than is TST. ${ }^{26,68}$ Because the risk of developing TB is increased in patients with IBD taking immunosuppressive therapy, increasing the sensitivity may be more important than a slight decrease in the specificity for detecting LTBI. Therefore, the use of TST alone is not appropriate for detecting LTBI in patients with IBD receiving immunosuppressive therapy prior to the initiation of anti-TNF therapy.

The "either test positive" strategy is a valid method for diagnosing LTBI; however, its superiority to other strategies is unclear

- Quality of evidence, moderate; recommendations, strong

- Level of agreement: strongly agree 17\%, agree 63\%, uncertain 16\%, disagree 4\%

Since TB infection in patients with IBD receiving antiTNF therapy may be severe, disseminated, and occasionally fatal, most experts agree that increasing sensitivity is more important than a slight decrease in specificity for detecting LTBI. ${ }^{34,54,87}$ The concomitant use of 2 or more immunosuppressive drugs may lead to false-negative results in both TST and IGRAs, and some authors recommend using both methods to increase the sensitivity of detecting LTBI in immunosuppressed patients prior to initiating anti-TNF therapy ${ }^{88,89}$

The recommended tests for diagnosing LTBI in immunosuppressed patients prior to initiating anti-TNF therapy may vary depending on the country. Some countries recommend TST first, followed by IGRAs in cases of a borderline or suspected false-negative TST or if a history of previous BCG vaccination is present. Others recommend the simultaneous use of TST and IGRAs for all cases. The Swiss Lung Association recommends that IGRAs are the only diagnostic test for LTBI $;^{90}$ however, TST alone is not recommended for detecting LTBI in most countries. Recently, the "either test positive" strategy (either test can be performed first, and if the test result is negative, then the other test can be performed) has 


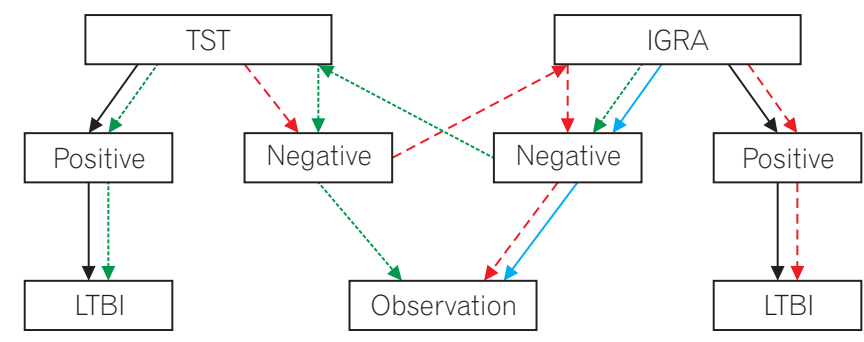

Patients with IBD with no history of tuberculosis treatment/close contact and a normal chest $X$-ray

Fig. 1. Algorithm for the diagnosis of latent tuberculosis infection in patients with IBD. Blue arrow, if a history of BCG vaccination is present; red arrow, start with tuberculin skin test (TST); green arrow, start with interferon-gamma releasing assay (IGRA). LTBI, latent tuberculosis infection.

been reported to reduce the rate of TB development close to the incidence of the general population. ${ }^{64}$ Therefore, we propose that the "either test positive" strategy is a valid method for diagnosing LTBI, although it is unclear whether it is superior to other strategies (Fig. 1).

\section{SUMMARY}

In summary, Part 1 of the Asian Organization for Crohn's and Colitis and Asia Pacific Association of Gastroenterology consensus on TB infection in patients with IBD receiving anti-TNF treatment highlighted the methodology in the development of the consensus statements, substantially higher risk of TB reactivation during anti-TNF therapy in many Asian countries, and various diagnostic tests for LTBI. Part 2 of the consensus statements highlight management of latent TB in preparation for anti-TNF therapy, monitoring during anti-TNF therapy, and management of an active TB infection after anti-TNF therapy.

\section{FINANCIAL SUPPORT}

This study was supported by a grant from the Korean Health Technology R\&D Project, Ministry of Health \& Welfare, Republic of Korea (A120176).

\section{CONFLICT OF INTEREST}

No potential conflict of interest relevant to this article was reported.

\section{AUTHOR CONTRIBUTION}

D.I.P., collecting and interpreting data, drafting the manuscript; S.K.Y., planning and conducting study, redrafting the manuscript; T.H., M.C., S.C.N., C.J.O., S.C.W., R.B., I.N.H., Y.T.J., D.S.H., H.J.K., Z.R., K.W., J.Q., P.J.H., K.M., A.A., Y.S., K.S., M.W., T.H., and A.S.P., collecting and interpreting data, redrafting the manuscript.

All of authors has approved the final draft submitted.

\section{REFERENCES}

1. Getahun H, Matteelli A, Chaisson RE, Raviglione M. Latent Mycobacterium tuberculosis infection. N Engl J Med 2015;372:2127-2135.

2. Mack U, Migliori GB, Sester M, et al. LTBI: latent tuberculosis infection or lasting immune responses to M. tuberculosis? A TBNET consensus statement. Eur Respir J 2009;33:956-973.

3. Fallahi-Sichani M, El-Kebir M, Marino S, Kirschner DE, Linderman JJ. Multiscale computational modeling reveals a critical role for TNF-alpha receptor 1 dynamics in tuberculosis granuloma formation. J Immunol 201 1;186:3472-3483.

4. Roach DR, Bean AG, Demangel C, France MP, Briscoe H, Britton WJ. TNF regulates chemokine induction essential for cell recruitment, granuloma formation, and clearance of mycobacterial infection. J Immunol 2002;168:4620-4627.

5. Mohan VP, Scanga CA, Yu K, et al. Effects of tumor necrosis factor alpha on host immune response in chronic persistent tuberculosis: possible role for limiting pathology. Infect Immun 2001;69:1847-1855.

6. Rutgeerts P, Van Assche G, Vermeire S. Review article: infliximab therapy for inflammatory bowel disease. Seven years on. Aliment Pharmacol Ther 2006;23:451-463.

7. Wallis RS, Broder MS, Wong JY, Hanson ME, Beenhouwer DO. Granulomatous infectious diseases associated with tumor necrosis factor antagonists. Clin Infect Dis 2004;38:1261-1265.

8. Tubach F, Salmon D, Ravaud P, et al. Risk of tuberculosis is higher with anti-tumor necrosis factor monoclonal antibody therapy than with soluble tumor necrosis factor receptor therapy: the three-year prospective French Research Axed on Tolerance of Biotherapies registry. Arthritis Rheum 2009;60:1884-1894.

9. Winthrop KL, Baxter R, Liu L, et al. Mycobacterial diseases and antitumour necrosis factor therapy in USA. Ann Rheum Dis 2013;72:37-42.

10. Navarra SV, Tang B, Lu L, et al. Risk of tuberculosis with anti-tumor necrosis factor-alpha therapy: substantially higher number of patients at risk in Asia. Int J Rheum Dis 2014;17:291-298. 
11. Weng MT, Wei SC, Lin CC, et al. Seminar report from the 2014 Taiwan Society of Inflammatory Bowel Disease (TSIBD) Spring Forum (May 24th, 2014): Crohn's disease versus intestinal tuberculosis infection. Intest Res 2015;13:6-10.

12. Song HK, Lee KM, Jung SA, et al. Quality of care in inflammatory bowel disease in Asia: the results of a multinational webbased survey in the 2(nd) Asian Organization of Crohn's and Colitis (AOCC) meeting in Seoul. Intest Res 2016;14:240-247.

13. Carmona L, Gomez-Reino JJ, Rodriguez-Valverde V, et al. Effectiveness of recommendations to prevent reactivation of latent tuberculosis infection in patients treated with tumor necrosis factor antagonists. Arthritis Rheum 2005;52:1766-1772.

14. Rahier JF, Ben-Horin S, Chowers Y, et al. European evidencebased consensus on the prevention, diagnosis and management of opportunistic infections in inflammatory bowel disease. J Crohns Colitis 2009;3:47-91.

15. Rahier JF, Magro F, Abreu C, et al. Second European evidencebased consensus on the prevention, diagnosis and management of opportunistic infections in inflammatory bowel disease. J Crohns Colitis 2014;8:443-468.

16. Getahun H, Matteelli A, Abubakar I, et al. Management of latent Mycobacterium tuberculosis infection: WHO guidelines for low tuberculosis burden countries. Eur Respir J 2015;46:1563-1576.

17. Favalli EG, Caporali R, Sinigaglia L, et al. Recommendations for the use of biologic therapy in rheumatoid arthritis: update from the Italian Society for Rheumatology II. Safety. Clin Exp Rheumatol 2011;29(3 Suppl 66):S15-S27.

18. Prevention Committee of the Japanese Society for Tuberculosis; Treatment Committee of the Japanese Society for Tuberculosis. Treatment guidelines for latent tuberculosis infection. Kekkaku 2014;89:21-37.

19. Nordgaard-Lassen I, Dahlerup JF, Belard E, et al. Guidelines for screening, prophylaxis and critical information prior to initiating anti-TNF-alpha treatment. Dan Med J 2012;59:C4480.

20. Iannone F, Cantini F, Lapadula G. Diagnosis of latent tuberculosis and prevention of reactivation in rheumatic patients receiving biologic therapy: international recommendations. J Rheumatol Suppl 2014;91:41-46.

21. Carpio D, Jauregui-Amezaga A, de Francisco R, et al. Tuberculosis in anti-tumour necrosis factor-treated inflammatory bowel disease patients after the implementation of preventive measures: compliance with recommendations and safety of retreatment. J Crohns Colitis 2016;10:1186-1193.

22. Bombardier C, Hazlewood GS, Akhavan P, et al. Canadian Rheumatology Association recommendations for the pharmacological management of rheumatoid arthritis with traditional and biologic disease-modifying antirheumatic drugs: part II safety. J Rheumatol 2012;39:1583-1602.
23. Shim TS. Diagnosis and treatment of latent tuberculosis infection in patients with inflammatory bowel diseases due to initiation of anti-tumor necrosis factor therapy. Intest Res 2014;12:12-19.

24. Lorenzetti R, Zullo A, Ridola L, et al. Higher risk of tuberculosis reactivation when anti-TNF is combined with immunosuppressive agents: a systematic review of randomized controlled trials. Ann Med 2014;46:547-554.

25. British Thoracic Society Standards of Care Committee. BTS recommendations for assessing risk and for managing Mycobacterium tuberculosis infection and disease in patients due to start anti-TNF-alpha treatment. Thorax 2005;60:800-805.

26. Solovic I, Sester M, Gomez-Reino JJ, et al. The risk of tuberculosis related to tumour necrosis factor antagonist therapies: a TBNET consensus statement. Eur Respir J 2010;36:1185-1206.

27. Chebli JM, Gaburri PD, Chebli LA, et al. A guide to prepare patients with inflammatory bowel diseases for anti-TNF-alpha therapy. Med Sci Monit 2014;20:487-498.

28. Joint Committee for the Revision of Korean Guidelines for Tuberculosis. Korean guidelines for tuberculosis. 2nd ed. Cheongju: Korean Centers for Disease Control and Prevention, 2014.

29. WHO handbook for guideline development. 2nd ed. Geneva: World Health Organization, 2014.

30. Guyatt GH, Oxman AD, Vist GE, et al. GRADE: an emerging consensus on rating quality of evidence and strength of recommendations. BMJ 2008;336:924-926.

31. Balshem H, Helfand M, Schunemann HJ, et al. GRADE guidelines: 3. rating the quality of evidence. J Clin Epidemiol 2011;64:401-406.

32. Guyatt GH, Oxman AD, Kunz R, et al. Going from evidence to recommendations. BMJ 2008;336:1049-1051.

33. Lee KM, Kim YS, Seo GS, Kim TO, Yang SK; IBD Study Group of the Korean Association for the Study of Intestinal Diseases. Use of thiopurines in inflammatory bowel disease: a consensus statement by the Korean Association for the Study of Intestinal Diseases (KASID). Intest Res 2015;13:193-207.

34. Keane J, Gershon S, Wise RP, et al. Tuberculosis associated with infliximab, a tumor necrosis factor alpha-neutralizing agent. N Engl J Med 2001;345:1098-1104.

35. Gomez-Reino JJ, Carmona L, Valverde VR, Mola EM, Montero MD; BIOBADASER Group. Treatment of rheumatoid arthritis with tumor necrosis factor inhibitors may predispose to significant increase in tuberculosis risk: a multicenter activesurveillance report. Arthritis Rheum 2003;48:2122-2127. 
36. Souto A, Maneiro JR, Salgado E, Carmona L, Gomez-Reino JJ. Risk of tuberculosis in patients with chronic immune-mediated inflammatory diseases treated with biologics and tofacitinib: a systematic review and meta-analysis of randomized controlled trials and long-term extension studies. Rheumatology (Oxford) 2014;53:1872-1885.

37. Cantini F, Niccoli L, Goletti D. Adalimumab, etanercept, infliximab, and the risk of tuberculosis: data from clinical trials, national registries, and postmarketing surveillance. J Rheumatol Suppl 2014;91:47-55.

38. Askling J, Fored CM, Brandt L, et al. Risk and case characteristics of tuberculosis in rheumatoid arthritis associated with tumor necrosis factor antagonists in Sweden. Arthritis Rheum 2005;52:1986-1992.

39. Ford AC, Peyrin-Biroulet L. Opportunistic infections with antitumor necrosis factor-alpha therapy in inflammatory bowel disease: meta-analysis of randomized controlled trials. Am J Gastroenterol 2013;108:1268-1276.

40. Jung SM, Ju JH, Park MS, et al. Risk of tuberculosis in patients treated with anti-tumor necrosis factor therapy: a nationwide study in South Korea, a country with an intermediate tuberculosis burden. Int J Rheum Dis 2015;18:323-330.

41. Dixon WG, Hyrich KL, Watson KD, et al. Drug-specific risk of tuberculosis in patients with rheumatoid arthritis treated with anti-TNF therapy: results from the British Society for Rheumatology Biologics Register (BSRBR). Ann Rheum Dis 2010;69:522528.

42. Global tuberculosis report 2013. World Health Organization Web site. http://www.who.int/tb/publications/global_report/ ed/. Accessed July 18, 2017.

43. Jauregui-Amezaga A, Turon F, Ordas I, et al. Risk of developing tuberculosis under anti-TNF treatment despite latent infection screening. J Crohns Colitis 2013;7:208-212.

44. Manosa M, Domenech E, Cabre E. Current incidence of active tuberculosis in IBD patients treated with anti-TNF agents: still room for improvement. J Crohns Colitis 2013;7:e499-e500. doi: 10.1016/j.crohns.2013.04.021.

45. Kisacik B, Pamuk ON, Onat AM, et al. Characteristics predicting tuberculosis risk under tumor necrosis factor-alpha inhibitors: report from a large multicenter cohort with high background prevalence. J Rheumatol 2016;43:524-529.

46. Kim ES, Song GA, Cho KB, et al. Significant risk and associated factors of active tuberculosis infection in Korean patients with inflammatory bowel disease using anti-TNF agents. World J Gastroenterol 2015;21:3308-3316.
47. Byun JM, Lee CK, Rhee SY, et al. Risks for opportunistic tuberculosis infection in a cohort of 873 patients with inflammatory bowel disease receiving a tumor necrosis factor-alpha inhibitor. Scand J Gastroenterol 2015;50:312-320.

48. Chang CW, Wei SC, Chou JW, et al. Safety and efficacy of adalimumab for patients with moderate to severe Crohn's disease: the Taiwan Society of Inflammatory Bowel Disease (TSIBD) Study. Intest Res 2014;12:287-292.

49. Tam LS, Leung CC, Ying SK, et al. Risk of tuberculosis in patients with rheumatoid arthritis in Hong Kong: the role of TNF blockers in an area of high tuberculosis burden. Clin Exp Rheumatol 2010;28:679-685.

50. Deepak P, Stobaugh DJ, Ehrenpreis ED. Infectious complications of TNF-alpha inhibitor monotherapy versus combination therapy with immunomodulators in inflammatory bowel disease: analysis of the Food and Drug Administration Adverse Event Reporting System. J Gastrointestin Liver Dis 2013;22:269276.

51. Abreu C, Magro F, Santos-Antunes J, et al. Tuberculosis in antiTNF-alpha treated patients remains a problem in countries with an intermediate incidence: analysis of 25 patients matched with a control population. J Crohns Colitis 2013;7:e486-e492. doi: 10.1016/j.crohns.2013.03.004.

52. Singh J, Puri AS, Sachdeva S, Sakhuja P, Arivarasan K. Rectal tuberculosis after infliximab therapy despite negative screening for latent tuberculosis in a patient with ulcerative colitis. Intest Res 2016;14:183-186.

53. Hsia EC, Schluger N, Cush JJ, et al. Interferon-gamma release assay versus tuberculin skin test prior to treatment with golimumab, a human anti-tumor necrosis factor antibody, in patients with rheumatoid arthritis, psoriatic arthritis, or ankylosing spondylitis. Arthritis Rheum 2012;64:2068-2077.

54. Abitbol Y, Laharie D, Cosnes J, et al. Negative screening does not rule out the risk of tuberculosis in patients with inflammatory bowel disease undergoing anti-TNF treatment: a descriptive study on the GETAID cohort. J Crohns Colitis 2016;10:11791185.

55. Kim HC, Jo KW, Jung YJ, et al. Diagnosis of latent tuberculosis infection before initiation of anti-tumor necrosis factor therapy using both tuberculin skin test and QuantiFERON-TB Gold In Tube assay. Scand J Infect Dis 2014;46:763-769.

56. Tanabe Seiyaku. Information for proper use of infliximab (in Japanese). Accessed March 29, 2017.

57. Vaughn BP, Doherty GA, Gautam S, Moss AC, Cheifetz AS. Screening for tuberculosis and hepatitis B prior to the initiation of anti-tumor necrosis therapy. Inflamm Bowel Dis 2012;18:1057-1063. 
58. Ponce de Leon D, Acevedo-Vasquez E, Alvizuri S, et al. Comparison of an interferon-gamma assay with tuberculin skin testing for detection of tuberculosis (TB) infection in patients with rheumatoid arthritis in a TB-endemic population. J Rheumatol 2008;35:776-781.

59. Diel R, Loddenkemper R, Nienhaus A. Evidence-based comparison of commercial interferon-gamma release assays for detecting active TB: a metaanalysis. Chest 2010;137:952-968.

60. American Thoracic Society; Centers for Disease Control and Prevention; Infectious Diseases Society of America. American Thoracic Society/Centers for Disease Control and Prevention/ Infectious Diseases Society of America: controlling tuberculosis in the United States. Am J Respir Crit Care Med 2005;172:11691227.

61. Menzies D, Pai M, Comstock G. Meta-analysis: new tests for the diagnosis of latent tuberculosis infection: areas of uncertainty and recommendations for research. Ann Intern Med 2007;146:340-354.

62. Matulis G, Juni P, Villiger PM, Gadola SD. Detection of latent tuberculosis in immunosuppressed patients with autoimmune diseases: performance of a Mycobacterium tuberculosis antigen-specific interferon gamma assay. Ann Rheum Dis 2008;67:84-90.

63. Mazurek GH, Jereb J, Vernon A, et al. Updated guidelines for using interferon gamma release assays to detect Mycobacterium tuberculosis infection: United States, 2010. MMWR Recomm Rep 2010;59:1-25

64. Jung YJ, Lee JY, Jo KW, et al. The 'either test positive' strategy for latent tuberculous infection before anti-tumour necrosis factor treatment. Int J Tuberc Lung Dis 2014;18:428-434.

65. Canadian Agency for Drugs and Technologies in Health (CADTH). Interferon-gamma release assays testing versus tuberculosis skin testing for tuberculosis: a review of the clinical effectiveness and guidelines. Rapid response report 2011. Ottawa: CADTH, 2011.

66. Singanayagam A, Manalan K, Sridhar S, et al. Evaluation of screening methods for identification of patients with chronic rheumatological disease requiring tuberculosis chemoprophylaxis prior to commencement of TNF-alpha antagonist therapy. Thorax 2013;68:955-961.

67. Reichmann MT, Marshall BG, Cummings F, Elkington PT. Tuberculosis and TNF-inhibitors: history of exposure should outweigh investigations. BMJ Case Rep 2014;2014:bcr2013202127. doi: 10.1136/bcr-2013-202127.

68. Qumseya BJ, Ananthakrishnan AN, Skaros S, et al. QuantiFERON TB gold testing for tuberculosis screening in an inflammatory bowel disease cohort in the United States. Inflamm Bowel Dis 2011;17:77-83.
69. Diel R, Loddenkemper R, Meywald-Walter K, Niemann S, Nienhaus A. Predictive value of a whole blood IFN-gamma assay for the development of active tuberculosis disease after recent infection with Mycobacterium tuberculosis. Am J Respir Crit Care Med 2008;177:1164-1170.

70. Bonfiglioli KR, Ribeiro AC, Moraes JC, et al. LTBI screening in rheumatoid arthritis patients prior to anti-TNF treatment in an endemic area. Int J Tuberc Lung Dis 2014;18:905-911.

71. Singh JA, Furst DE, Bharat A, et al. 2012 update of the 2008 American College of Rheumatology recommendations for the use of disease-modifying antirheumatic drugs and biologic agents in the treatment of rheumatoid arthritis. Arthritis Care Res (Hoboken) 2012;64:625-639.

72. Grzybowski S, Fishaut H, Rowe J, Brown A. Tuberculosis among patients with various radiologic abnormalities, followed by the chest clinic service. Am Rev Respir Dis 1971;104:605-608.

73. Efficacy of various durations of isoniazid preventive therapy for tuberculosis: five years of follow-up in the IUAT trial. International Union against Tuberculosis Committee on Prophylaxis. Bull World Health Organ 1982;60:555-564.

74. Targeted tuberculin testing and treatment of latent tuberculosis infection. This official statement of the American Thoracic Society was adopted by the ATS Board of Directors, July 1999. This is a Joint Statement of the American Thoracic Society (ATS) and the Centers for Disease Control and Prevention (CDC). This statement was endorsed by the Council of the Infectious Diseases Society of America. (IDSA), September 1999, and the sections of this statement. Am J Respir Crit Care Med 2000;161:S221-S247.

75. Horsburgh CR Jr. Priorities for the treatment of latent tuberculosis infection in the United States. N Engl J Med 2004;350:20602067.

76. Araujo Z, de Waard JH, de Larrea CF, Borges R, Convit J. The effect of bacille Calmette-Guerin vaccine on tuberculin reactivity in indigenous children from communities with high prevalence of tuberculosis. Vaccine 2008;26:5575-5581.

77. Chan PC, Chang LY, Wu YC, et al. Age-specific cut-offs for the tuberculin skin test to detect latent tuberculosis in BCG-vaccinated children. Int J Tuberc Lung Dis 2008;12:1401-1406.

78. Diel R, Loddenkemper R, Niemann S, Meywald-Walter K, Nienhaus A. Negative and positive predictive value of a whole-blood interferon-gamma release assay for developing active tuberculosis: an update. Am J Respir Crit Care Med 2011;183:88-95.

79. Shahidi N, Fu YT, Qian H, Bressler B. Performance of interferongamma release assays in patients with inflammatory bowel disease: a systematic review and meta-analysis. Inflamm Bowel Dis 2012;18:2034-2042. 
80. Mow WS, Abreu-Martin MT, Papadakis KA, Pitchon HE, Targan SR, Vasiliauskas EA. High incidence of anergy in inflammatory bowel disease patients limits the usefulness of PPD screening before infliximab therapy. Clin Gastroenterol Hepatol 2004;2:309-313.

81. Schoepfer AM, Flogerzi B, Fallegger S, et al. Comparison of interferon-gamma release assay versus tuberculin skin test for tuberculosis screening in inflammatory bowel disease. Am J Gastroenterol 2008;103:2799-2806.

82. Jasmer RM, Nahid P, Hopewell PC. Clinical practice: latent tuberculosis infection. N Engl J Med 2002;347:1860-1866.

83. Pai M, Riley LW, Colford JM Jr. Interferon-gamma assays in the immunodiagnosis of tuberculosis: a systematic review. Lancet Infect Dis 2004;4:761-776.

84. Papay P, Eser A, Winkler S, et al. Factors impacting the results of interferon-gamma release assay and tuberculin skin test in routine screening for latent tuberculosis in patients with inflammatory bowel diseases. Inflamm Bowel Dis 2011;17:84-90.
85. Ferrara G, Losi M, Meacci M, et al. Routine hospital use of a new commercial whole blood interferon-gamma assay for the diagnosis of tuberculosis infection. Am J Respir Crit Care Med 2005;172:631-635.

86. Wong SH, Ip M, Tang W, et al. Performance of interferon-gamma release assay for tuberculosis screening in inflammatory bowel disease patients. Inflamm Bowel Dis 2014;20:2067-2072.

87. Sichletidis L, Settas L, Spyratos D, Chloros D, Patakas D. Tuberculosis in patients receiving anti-TNF agents despite chemoprophylaxis. Int J Tuberc Lung Dis 2006;10:1127-1132.

88. Cotter J, Rosa B. The importance of IGRA in patients candidates for biological therapy. J Crohns Colitis 2013;7:928-929.

89. Duarte R, Campainha S, Cotter J, et al. Position paper on tuberculosis screening in patients with immune mediated inflammatory diseases candidates for biological therapy. Acta Reumatol Port 2012;37:253-259.

90. Beglinger C, Dudler J, Mottet C, et al. Screening for tuberculosis infection before the initiation of an anti-TNF-alpha therapy. Swiss Med Wkly 2007;137:620-622. 